\section{SOI: $1.1 / \mathrm{TAS}$
International Scientific Journal Theoretical \& Applied Science}

p-ISSN: 2308-4944 (print)

e-ISSN: 2409-0085 (online)

Year: 2017

Issue: 04

Volume: 48

Published: 30.04 .2017

http://T-Science.org
Normahmad Ravshanov

Doctor of Sciences, Head of Laboratory, Tashkent University of information Technologies,

Tashkent, Uzbekistan

Farruh Muradov

Staff researcher,

Tashkent University of information Technologies,

Tashkent, Uzbekistan

SECTION 2. Applied mathematics.

Mathematical modeling.

\title{
MODELS AND NUMERICAL ALGORITHMS OF ACTIVE TRANSPORT AND DIFFUSION OF FINE PARTICLES IN THE ATMOSPHERE
}

\begin{abstract}
In the article importance of the solving the problem of monitoring and forecasting of the ecological status of industrial regions is observed, where has an imbalance of sanitary environmental regulations due to the large number of emissions of harmful active fine aerosol particles and carbon dioxide gases into the atmosphere. A mathematical model of the spread of pollutants released into the environment from production facilities is displayed, which describes the system of differential equations in partial derivatives with appropriate initial and boundary conditions. The main parameters that play a significant role in the transport and diffusion of pollutants in the atmosphere: the wind speed and direction; terrain; absorption coefficient harmful aerosol of fine particles in the atmosphere, etc is defined. We obtain the differential equation for calculating the rate of deposition of fine particulate and propagating in the boundary layer of the atmosphere, when the principal parameters affecting the rate of particle deposition the mass and radius of the aerosol particle density of the atmosphere, air resistance force are considered. Efficient numerical algorithm is proposed for the numerical solution of the problem, based on the "direct method". The algorithm allows to reduce a multidimensional problem described by differential equations in partial derivatives to the integration of ordinary differential equations.

Key words: mathematical model, transfer and diffusion of pollutants, climatic factor, fluid mechanics, numerical algorithm.

Language: English

Citation: Ravshanov N, Muradov F (2017) MODELS AND NUMERICAL ALGORITHMS OF ACTIVE TRANSPORT AND DIFFUSION OF FINE PARTICLES IN THE ATMOSPHERE. ISJ Theoretical \& Applied Science, 04 (48): 189-197.

Soi: http://s-o-i.org/1.1/TAS-04-48-30 Doi: crossef https://dx.doi.org/10.15863/TAS.2017.04.48.30
\end{abstract}

\section{Introduction}

Construction and commissioning of industrial facilities and increase their capacity, without taking into account the sanitary norms, the development and introduction of the new oil and gas fields, extraction of ores from the bottom of the earth, increasing of the transport systems in cities and metropolitan areas, etc. violate the ecological balance of the region and the surrounding area. The imbalance is caused by increasing the gas content of the atmosphere and the concentration of harmful fine particles in its surface layer. These "negative" effects adversely affect to the living system - the flora and fauna of the region and at the global level are making contribution to climate change in the world.

As the International Committee for Health noted - the number of cases of cancer has risen dramatically over the past few years, asthma, allergies, etc. diseases due to deteriorating state of the environment throughout the world.
As ITAR-TASS reports for the month of December 2016 in China, France, Mongolia, the Balkans and other regions have been declared critical levels of air pollution. In particular, in Beijing for the elimination of environmental threats to measures included a cause to stop for a few days of the industrial facilities, lessons in schools, childcare institutions, and restrictions on the movement of vehicles inside the city.

Based on the above, the issues of monitoring, prediction and assessment of air pollution and the underlying land surface with passive and active aerosol emissions and fine impurities; Accommodation of the industrial enterprises in compliance with health standards; determine the amount of particulate matter is relevant in the environmental problems of the region and spread into the surrounding condition. 


\begin{tabular}{|c|c|c|c|c|c|c|}
\hline Impact Factor: & $\begin{array}{l}\text { ISRA (India) } \\
\text { ISI (Dubai, UAF } \\
\text { GIF (Australia) } \\
\text { JIF }\end{array}$ & $\begin{array}{l}=1.344 \\
=0.829 \\
=0.564 \\
=1.500\end{array}$ & $\begin{array}{l}\text { SIS (USA) } \\
\text { PИНЦ (Russia) } \\
\text { ESJI (KZ) } \\
\text { SJIF (Morocco) }\end{array}$ & $\begin{array}{l}=0.912 \\
=0.234 \\
=3.860 \\
=\mathbf{2 . 0 3 1}\end{array}$ & $\begin{array}{l}\text { ICV (Poland) } \\
\text { PIF (India) } \\
\text { IBI (India) }\end{array}$ & $\begin{array}{l}=6.630 \\
=1.940 \\
=4.260\end{array}$ \\
\hline
\end{tabular}

\section{Review of Literature}

The problems of mathematical modeling of the transport processes, diffusion and transport of hazardous substances (carbon dioxide gases, fine aerosol particles of active and passive) are engaged in scientific schools created under the direction of GI Marchuk VV Penenko, AE Aloyan, L.T. Matveeva, VP Dymnikova IE Naatsa, EA Zakarin, IA Kibel, LN Gutmann, FB Abutalieva, as well as foreign scientists W.J. Layton, J.H. Ferziger, J.W. Deardorff, M. Germano, U. Piomelli, L.C. Berselli, G.S. Winckelmans, W.C. Reynolds, H. Zidisk, KA Velds, KI Nappa, J. Gotaas, Myullioland M., S. ladder, M. Mathiesen, V. Edelman et al.

Developed under the leadership of their mathematical tools are widely used in monitoring, forecasting and assessment of the impact of anthropogenic impacts on the environment.

In the derivation of mathematical models of the above-mentioned objects of research scientists took as a basis the fundamental laws of hydrodynamics, mass and energy conservation of momentum and movement.

An important contribution made in this direction AA Samara, AA Tikhonov, GI Marchuk, P. topics VV Penenko, AE Aloyan and others. New effective numerical algorithms for computational experiments and solve problems on a computer were suggested by them.

In particular, in [1-3] developed mathematical models and numerical algorithms and software for predicting and monitoring the movement of a multicomponent air and transport of pollutants in the atmosphere, and also solved the problem of motion of a multicomponent air pollution in the atmosphere, taking into account of evaporation and condensation.

The paper [4] is devoted to the study of the migration process and the diffusion of active aerosol particles in the atmosphere, taking into account the chemical reactions in the air. Highlights chemical reactions occurring with the aerosol particles in the atmosphere.

In [5] studied the processes of transformation of substances in the process of transport and diffusion of pollutants in the air for long and medium distances. The methods of measurements and concentration of aerosol particles in the atmosphere, emitted from various sources and participating in long-range transport are nominated. The paper also explores the evolutionary trajectory and propagation models of aerosol particles in the atmosphere and set out a comparison of simulation results with in situ measurements.

For a description of the physical transport process of suspended particles in the atmosphere, there are also a number of works, which set out different approaches. These include methods for process studies using statistical models based on Gaussian distribution function [6-8].
In [9] considered the problems and principles of mathematical modeling, numerical algorithms and software macroscale physical processes in the atmosphere. The paper describes the modeling of atmospheric pollution biogas emitted from the decomposition of waste.

In [10] judged the transient process of transport of harmful bioaerosol substances given particle size, and physical processes of condensation and evaporation of gaseous substance.

The paper [11] devoted to the development of mathematical models of the dynamics of a two-speed granular medium, including the phase equilibrium temperature and pressure in the absence of phase equilibrium. The authors evaluated the ecological condition of air oil and gas field. Evaluation is done by continuous air monitoring, obtained by automatic environmental control post. According to this deposit 14 measured parameters: the concentration of hydrocarbons, nitrogen oxides, carbon monoxide, ozone, weather parameters, such as temperature, pressure, wind velocity, exposure dose, and the oxygen content in the ambient air.

In [12-19] developed a mathematical model to calculate and predict the transport of pollutants in the industrial zone Akhangaran Uzbekistan; local hydrodynamic model and design scheme spread of pollutants in the atmosphere for industrial region; three-dimensional numerical model for the assessment of air pollution Akhangaran Valley industrial emissions of sulfur dioxide (SO2) and arsenic (As); a new approach that allows to use experimental data on the wind conditions in one of the valleys of the Western Tien Shan to other valleys with similar morphometric characteristics. The authors estimated the state of the air basin Akhangaran valley for different types of circulation in the warm and the cold half of the year; installed wind speeds, providing favorable and unfavorable conditions of purification of the atmosphere from harmful emissions in the Valley; found an association of favorable and unfavorable conditions for air purification with the types of synoptic processes in Central Asia; proposed monitoring scheme, which includes a short-term forecast of air basin.

Investigation of the process of distribution of harmful substances into the environment showed that the main factors which directly affect to the course of this process: the speed of movement of air masses in the atmosphere; the diffusion coefficient and the coefficient of turbulent mixing in the vertical; wind rose over the time, and depending on the orography of the terrain; accounting the phase transition substance by changing the temperature in the atmosphere, as well as the rate of deposition of fine particulate matter and propagating in the boundary layer of the atmosphere.

Considering the above mentioned factors, operating in the process of transfer and diffusion of 


\begin{tabular}{|c|c|c|c|c|c|c|}
\hline Impact Factor: & $\begin{array}{l}\text { ISRA (India) } \\
\text { ISI (Dubai, UAF } \\
\text { GIF (Australia) } \\
\text { JIF }\end{array}$ & $\begin{array}{l}=1.344 \\
=0.829 \\
=0.564 \\
=1.500\end{array}$ & $\begin{array}{l}\text { SIS (USA) } \\
\text { PИНЦ (Russia) } \\
\text { ESJI (KZ) } \\
\text { SJIF (Morocco) }\end{array}$ & $\begin{array}{l}=0.912 \\
=0.234 \\
=3.860 \\
=\mathbf{2 . 0 3 1}\end{array}$ & $\begin{array}{l}\text { ICV (Poland) } \\
\text { PIF (India) } \\
\text { IBI (India) }\end{array}$ & $\begin{array}{l}=6.630 \\
=1.940 \\
=4.260\end{array}$ \\
\hline
\end{tabular}

harmful emissions into the atmosphere is necessary to develop an effective tool easily implemented - model, numerical algorithm, software and instrumentation system for monitoring, forecasting and management decision-making to prevent negative consequences for the environment.

\section{Problem Statement}

In order to study and predict the spread of the process, aerosol emissions, taking into account the above factors, the mathematical model of the object described by the transfer and diffusion equation and based on the law of conservation of mass, momentum:

$$
\begin{aligned}
& \frac{\partial \theta_{1}}{\partial t}+\left(w-w_{g}\right) \frac{\partial \theta_{1}}{\partial z}= \\
& =\mu_{1}\left(\frac{\partial^{2} \theta_{1}}{\partial x^{2}}+\frac{\partial^{2} \theta_{1}}{\partial y^{2}}\right)+\frac{\partial}{\partial z}\left(k \frac{\partial \theta_{1}}{\partial z}\right)+ \\
& +\delta Q_{1}+F_{1}+\Phi_{1} ; \\
& \frac{\partial \theta_{2}}{\partial t}+\left(w-w_{g}\right) \frac{\partial \theta_{2}}{\partial z}= \\
& =\mu_{1}\left(\frac{\partial^{2} \theta_{2}}{\partial x^{2}}+\frac{\partial^{2} \theta_{2}}{\partial y^{2}}\right)+\frac{\partial}{\partial z}\left(k \frac{\partial \theta_{2}}{\partial z}\right)+ \\
& +\delta Q_{2}+F_{2}+\Phi_{2} ; \\
& \frac{d w_{g}}{d t}=\frac{m g-6 \pi k r w_{g}-0.5 c \rho S w_{g}^{2}}{m} \text {; } \\
& \theta_{1}(x, y, z, \dot{0})=\theta_{1}^{0}(x, y, z) \text {; } \\
& \theta_{2}=\theta_{2}^{0}(x, y, z) \text {; } \\
& w_{g}(0)=w_{g}^{0} \text { при } t=0 \text {; } \\
& \left.\frac{\partial \theta_{1}}{\partial x}\right|_{x=0}=0 ;\left.\quad \frac{\partial \theta_{1}}{\partial x}\right|_{x=L_{x}}=0 ; \\
& \left.\frac{\partial \theta_{1}}{\partial y}\right|_{y=0}=0 ;\left.\quad \frac{\partial \theta_{1}}{\partial y}\right|_{y=L_{y}}=0 ; \\
& \left.\frac{\partial \theta_{1}}{\partial z}\right|_{z=0}=\eta_{1} \theta_{1} ;\left.\quad \frac{\partial \theta_{1}}{\partial z}\right|_{z=L_{z}}=0 \\
& \left.\frac{\partial \theta_{2}}{\partial x}\right|_{x=0}=0 ;\left.\quad \frac{\partial \theta_{2}}{\partial x}\right|_{x=L_{x}}=0 \text {; } \\
& \left.\frac{\partial \theta_{2}}{\partial y}\right|_{y=0}=0 ;\left.\quad \frac{\partial \theta_{2}}{\partial y}\right|_{y=h y}=0 \text {; } \\
& \left.\frac{\partial \theta_{2}}{\partial z}\right|_{z=0}=\eta_{2} \theta_{2} ;\left.\quad \frac{\partial \theta_{2}}{\partial z}\right|_{z=L_{z}}=0,
\end{aligned}
$$

where

$$
\begin{aligned}
& F_{1}^{n+1}=\left(\alpha_{2} \theta_{2}-\beta_{1} \theta_{1}\right) ; \Phi_{1}^{n}=u \frac{\partial \theta_{1}^{n}}{\partial x}+v \frac{\partial \theta_{1}^{n}}{\partial y} \\
& F_{2}^{n+1}=\left(\alpha_{1} \theta_{1}-\beta_{2} \theta_{2}\right) ; \Phi_{2}^{n}=u \frac{\partial \theta_{2}^{n}}{\partial x}+v \frac{\partial \theta_{2}^{n}}{\partial y} .
\end{aligned}
$$

Here $\theta_{1}, \theta_{2}$ - consentration of the spread of the first and second components of harmful substanses in atmosphere, $t$ - time, $x, y, z$ - coordinations, $u, v, w$ $x, y, z$ composed speed of wind by directions $\mathrm{x}, \mathrm{y}, \mathrm{z}$ respectivly, $w_{g}$ - speed of the spread particle , $k$ coefficient of turbulent mixing, $\mu$ - coefficient of diffusion, $\alpha_{i}, \beta_{i}$ - speed of transformation admixture i- into $\mathrm{i}-1$ and $i+1$ type $(\mathrm{i}=1,2) \eta_{1}, \eta_{2}$ - coefficient of cooperation with littering surface, $Q_{i}(x, y, z, t)$ power of the source of the first and second components, $\mathrm{m}$ - mass of the aerosol particle, $\mathrm{r}$ radius of particle, $\mathrm{c}$ - dimensionless quantity equal $0.5, \rho$ - consistense of atmosphere.

Methods for solving the problem. Since the problem (1) - (7) describes multidimensional differential equations in partial derivatives, to obtain its solution in analytical form is difficult. To solve the problem using semi-implicit finite-difference scheme in time and get a [20-22]:

$$
\begin{gathered}
\frac{\theta_{1}^{n+1}-\theta_{1}^{n}}{\Delta t}+\left(w-w_{g}^{n+1}\right) \frac{\partial \theta_{1}^{n+1}}{\partial z}=\mu_{1}\left(\frac{\partial^{2} \theta_{1}}{\partial x^{2}}+\frac{\partial^{2} \theta_{1}}{\partial y^{2}}\right)^{n+1}+ \\
+\frac{\partial}{\partial z}\left(k \frac{\partial \theta_{1}}{\partial z}\right)^{n+1}+\delta Q_{1}^{n+1}+F_{1}^{n+1}+\Phi_{1}^{n} ; \\
\frac{\theta_{2}^{n+1}-\theta_{2}^{n}}{\Delta t}+\left(w-w_{g}^{n+1}\right) \frac{\partial \theta_{2}^{n+1}}{\partial z}=\mu_{1}\left(\frac{\partial^{2} \theta_{2}}{\partial x^{2}}+\frac{\partial^{2} \theta_{2}}{\partial y^{2}}\right)^{n+1}+ \\
+\frac{\partial}{\partial z}\left(k \frac{\partial \theta_{2}}{\partial z}\right)^{n+1}+\delta Q_{2}^{n+1}+F_{2}^{n+1}+\Phi_{2}^{n} ; \\
\frac{\theta_{1}^{n+1}-\theta_{1}^{n}}{\Delta t}+\left(w-w_{g}^{n+1}\right) \frac{\partial \theta_{1}^{n+1}}{\partial z}= \\
=\mu_{1}\left(\frac{\partial^{2} \theta_{1}}{\partial x^{2}}+\frac{\partial^{2} \theta_{1}}{\partial y^{2}}\right)^{n+1}+\frac{\partial}{\partial z}\left(k \frac{\partial \theta_{1}}{\partial z}\right)^{n+1}+ \\
+\delta Q_{1}^{n+1}+F_{1}^{n+1}+\Phi_{1}^{n} ; \\
\frac{\theta_{2}^{n+1}-\theta_{2}^{n}}{\Delta t}+\left(w-w_{g}^{n+1}\right) \frac{\partial \theta_{2}^{n+1}}{\partial z}= \\
=\mu_{1}\left(\frac{\partial^{2} \theta_{2}}{\partial x^{2}}+\frac{\partial^{2} \theta_{2}}{\partial y^{2}}\right)^{n+1}+\frac{\partial}{\partial z}\left(k \frac{\partial \theta_{2}}{\partial z}\right)^{n+1}+ \\
+\delta Q_{2}^{n+1}+F_{2}^{n+1}+\Phi_{2}^{n} .
\end{gathered}
$$

Since the equation (3) is non-linear, for its decision is used an iterative method

$$
\frac{w_{g}^{n+1}-w_{g}^{n}}{\Delta t}=\frac{m g-6 \pi k r w_{g}^{n}-0.5 \cos \left(2 w_{g} w_{g}^{n}-w_{g}^{2}\right)}{m}
$$

and the conditions for the convergence of the iterative process is as follows:

$$
\left|w_{g}^{(s+1)}-w_{g}^{(s)}\right| \leq \varepsilon,
$$




\begin{tabular}{|c|c|c|c|c|c|c|}
\hline Impact Factor: & $\begin{array}{l}\text { ISRA (India) } \\
\text { ISI (Dubai, UAE } \\
\text { GIF (Australia) } \\
\text { JIF }\end{array}$ & $\begin{array}{l}=1.344 \\
=0.829 \\
=0.564 \\
=1.500\end{array}$ & $\begin{array}{l}\text { SIS (USA) } \\
\text { PИНЦ (Russia) } \\
\text { ESJI (KZ) } \\
\text { SJIF (Morocco) }\end{array}$ & $\begin{array}{l}=0.912 \\
=0.234 \\
=\mathbf{3 . 8 6 0} \\
=\mathbf{2 . 0 3 1}\end{array}$ & $\begin{array}{l}\text { ICV (Poland) } \\
\text { PIF (India) } \\
\text { IBI (India) }\end{array}$ & $\begin{array}{l}=6.630 \\
=1.940 \\
=4.260\end{array}$ \\
\hline
\end{tabular}

where $\varepsilon$ set size of the iterations, $\left(10^{-6}\right), s$ - quantity of the iterations.

\section{Solution Method}

From the formulation of the problem (1) - (7) it is seen that the coefficients of equation (1) - (2) does not depend on $\mathrm{x}$ and $\mathrm{y}$, hence it is possible to use a method of direct integration [23].

For solving the problem introducing network by variable $\mathrm{x}$ and $\mathrm{y}$

$$
\begin{gathered}
\omega_{x}=\left(x_{i}=i h_{x}, i=0,1,3, \mathrm{~N}_{1}+1 ; h_{x}=\frac{L_{x}}{\left(\mathrm{~N}_{1}+1\right)}\right) ; \\
\omega_{y}=\left(y_{j}=j h_{y}, j=0,1,2,3, \mathrm{~N}_{2}+1 ; h_{y}=\frac{L_{y}}{\left(\mathrm{~N}_{2}+1\right)}\right) .
\end{gathered}
$$

Further putting down equation (1), (2)) in $x=x_{i}$ we get system of linear equation

$$
\begin{gathered}
\frac{1}{\Delta t} \theta_{1, i}+\left(w-w_{g}^{n+1}\right) \frac{\partial \theta_{1, i}}{\partial z}= \\
=\mu_{1} \frac{\theta_{1, i-1}-2 \theta_{1, i}+\theta_{1, i+1}}{h_{x}^{2}}+ \\
+\mu_{1} \frac{\partial^{2} \theta_{1, i}}{\partial y^{2}}+\frac{\partial}{\partial z}\left(k \frac{\partial \theta_{1, i}}{\partial z}\right)+ \\
+\delta_{i} Q_{1, i}+F_{1, i}+\Phi_{1, i}+\frac{1}{\Delta \tau} \bar{\theta}_{1, i} ; \\
\quad \frac{1}{\Delta t} \theta_{2, i}+\left(w-w_{g}\right) \frac{\partial \theta_{2, i}}{\partial z}= \\
=\mu_{1} \frac{\theta_{2, i-1}-2 \theta_{2, i}+\theta_{2, i+1}}{h_{x}^{2}}+ \\
+\mu_{1} \frac{\partial^{2} \theta_{2, i}}{\partial y^{2}}+\frac{\partial}{\partial z}\left(k \frac{\partial \theta_{2, i}}{\partial z}\right)+ \\
+\delta_{i} Q_{2, i}+F_{2, i}+\Phi_{2, i}+\frac{1}{\Delta \tau} \bar{\theta}_{2, i},
\end{gathered}
$$

where

$$
\begin{aligned}
& \Phi_{1, i}=\left[\frac{U+|U|}{2}\left(\bar{\theta}_{1, i}-\bar{\theta}_{1, i-1}\right) / h_{x} \mid+\frac{V+|V|}{2} \frac{\partial \bar{\theta}_{1}}{\partial y}\right] ; \\
& \Phi_{2, i}=\left[\frac{U+|U|}{2}\left(\bar{\theta}_{2, i}-\bar{\theta}_{2, i-1}\right) / h_{x} \mid+\frac{V+|V|}{2} \frac{\partial \bar{\theta}_{2}}{\partial y}\right] .
\end{aligned}
$$

For the convenience equation 8-9 at compact aspect might be written

$$
\begin{gathered}
\frac{1}{\Delta t} \theta_{1, i}+\left(w-w_{g}\right) \frac{\partial \theta_{1, i}}{\partial z}= \\
=\mu_{1} \frac{M_{1}}{h_{x}^{2}} \theta_{1, i}+\mu_{1} \frac{\partial^{2} \theta_{1, i}}{\partial y^{2}}+\frac{\partial}{\partial z}\left(k \frac{\partial \theta_{1, i}}{\partial z}\right)+ \\
+\delta_{i} Q_{1, i}+F_{1, i}+\Phi_{1, i}+\frac{1}{\Delta t} \bar{\theta}_{1, i} ;
\end{gathered}
$$

$$
\begin{gathered}
\frac{1}{\Delta t} \theta_{2, i}+\left(w-w_{g}\right) \frac{\partial \theta_{2, i}}{\partial z}= \\
=\mu_{1} \frac{M_{1}}{h_{x}^{2}} \theta_{2, i}+\mu_{1} \frac{\partial^{2} \theta_{2, i}}{\partial y^{2}}+\frac{\partial}{\partial z}\left(k \frac{\partial \theta_{2, i}}{\partial z}\right)+ \\
+\delta_{i} Q_{2, i}+F_{2, i}+\Phi_{2, i}+\frac{1}{\Delta t} \bar{\theta}_{2, i} .
\end{gathered}
$$

Here

$$
M_{1}=\left(\begin{array}{cccccc}
-2 & 1 & 0 & \ldots & \ldots & 0 \\
1 & -2 & 1 & \ldots & \ldots & 0 \\
0 & 1 & -2 & 1 & \ldots & \ldots \\
\ldots & \ldots & \ldots & \ldots & \ldots & \ldots \\
\ldots & \ldots & \ldots & \ldots & 1 & -2
\end{array}\right)
$$

Since the matrix $M_{1}$ is the matrix of the simple structure , it can be represented as $M_{1}=B_{1} \lambda_{1, i} B_{1}^{t}, \quad \lambda_{1, i}=B_{1}^{t} M_{1} B_{1}, \quad B_{1}^{-1}=B_{1}^{t}, \quad \lambda \quad$ is a diagonal matrix, which elemets are own sence of the matrix $M_{1}$

$$
\lambda_{1, i}=\left(\begin{array}{ccccc}
\lambda_{1} & 0 & 0 & 0 & \\
0 & \lambda_{2} & 0 & 0 & \\
0 & 0 & \lambda_{3} & 0 & \\
0 & 0 & \ldots & \ldots & \\
& & & & \\
0 & 0 & 0 & \ldots & \lambda_{N_{1}}
\end{array}\right),
$$

$\lambda_{1, i}$ and elements of $B_{1}$ matrix calculates with formulas:

$$
\begin{gathered}
\lambda_{1, i}=-2\left(1-\cos \frac{i \pi}{N_{1}+1}\right) ; \\
b_{1, i, j}=(-1) \sqrt[i+j]{\frac{2}{N_{1}+1}} \sin \frac{i j \pi}{N_{1}+1} ;\left(i, j=\overrightarrow{1} N_{1}\right) .
\end{gathered}
$$

Obtained equation (10) and (11), multiplying by matrix on the left $B_{1}^{t}$ and introducing sign $B_{1}^{t} \theta_{1}=\theta_{1}^{(1)}$ и $B_{1}^{t} \theta_{2}=\theta_{2}^{(1)}$ we get :

$$
\begin{aligned}
& \frac{1}{\Delta t} \theta_{1, i}^{(1)}+\left(w-w_{g}\right) \frac{\partial \theta_{1, i}^{(1)}}{\partial z}=\alpha_{i}^{2} \theta_{1, i}^{(1)}+\mu_{1} \frac{\partial^{2} \theta_{1, i}^{(1)}}{\partial y^{2}}+ \\
& +\frac{\partial}{\partial z}\left(k \frac{\partial \theta_{1, i}^{(1)}}{\partial z}\right)+\delta_{i} Q_{1, i}^{(1)}+F_{1, i}^{(1)}+\Phi_{1, i}^{(1)}+\frac{1}{\Delta t} \bar{\theta}_{1, i}^{(1)} \\
& \frac{1}{\Delta t} \theta_{2, i}^{(1)}+\left(w-w_{g}\right) \frac{\partial \theta_{2, i}^{(1)}}{\partial z}=\alpha_{1, i}^{2} \theta_{2, i}^{(1)}+\mu_{1} \frac{\partial^{2} \theta_{2, i}^{(1)}}{\partial y^{2}}+ \\
& +\frac{\partial}{\partial z}\left(k \frac{\partial \theta_{2, i}^{(1)}}{\partial z}\right)+\delta_{i} Q_{2, i}^{(1)}+F_{2, i}^{(1)}+\Phi_{2, i}^{(1)}+\frac{1}{\Delta t} \bar{\theta}_{2, i}^{(1)} \\
& \quad \text { Here } \alpha_{1, i}^{2}=\frac{\gamma_{1, i} \mu_{1}}{h_{x}^{2}}
\end{aligned}
$$




\begin{tabular}{|c|c|c|c|c|c|c|}
\hline Impact Factor: & $\begin{array}{l}\text { ISRA (India) } \\
\text { ISI (Dubai, UAE } \\
\text { GIF (Australia) } \\
\text { JIF }\end{array}$ & $\begin{array}{l}=1.344 \\
=0.829 \\
=0.564 \\
=1.500\end{array}$ & $\begin{array}{l}\text { SIS (USA) } \\
\text { PИНЦ (Russia) } \\
\text { ESJI (KZ) } \\
\text { SJIF (Morocco) }\end{array}$ & $\begin{array}{l}=0.912 \\
=0.234 \\
=\mathbf{3 . 8 6 0} \\
=\mathbf{2 . 0 3 1}\end{array}$ & $\begin{array}{l}\text { ICV (Poland) } \\
\text { PIF (India) } \\
\text { IBI (India) }\end{array}$ & $\begin{array}{l}=6.630 \\
=1.940 \\
=4.260\end{array}$ \\
\hline
\end{tabular}

Analogous at equations (12) and (13) differential operators by $\mathrm{y}$, replaced with ultimate- diverse,we obtain:

$$
\begin{aligned}
& \frac{1}{\Delta t} \theta_{1, i, j}^{(1)}+\left(w-w_{g}\right) \frac{\partial \theta_{1, i, j}^{(1)}}{\partial z}=\alpha_{1, i}^{2} \theta_{1, i, j}^{(1)}+\mu_{1} \frac{M_{1}}{h y^{2}} \theta_{1, i, j}^{(1)}+ \\
& +\frac{\partial}{\partial z}\left(k \frac{\partial \theta_{1, i, j}^{(1)}}{\partial z}\right)+\delta_{i j} Q_{1, i, j}^{(1)}+F_{1, i, j}^{(1)}+\Phi_{1, i, j}^{(1)}+\frac{1}{\Delta t} \bar{\theta}_{1, i, j}^{(1)} \\
& \frac{1}{\Delta t} \theta_{2, i, j}^{(1)}+\left(w-w_{g}\right) \frac{\partial \theta_{2, i, j}^{(1)}}{\partial z}=\alpha_{i}^{2} \theta_{2, i, j}^{(1)}+\mu_{1} \frac{M_{2}}{h y^{2}} \theta_{2, i, j}^{(1)}+ \\
& +\frac{\partial}{\partial z}\left(k \frac{\partial \theta_{2, i, j}^{(1)}}{\partial z}\right)+\delta_{i j} Q_{2, i, j}^{(1)}+F_{2, i, j}^{(1)}+\Phi_{2, i, j}^{(1)}+\frac{1}{\Delta t} \bar{\theta}_{2, i, j}^{(1)}
\end{aligned}
$$

The matrix $M_{2}$ also is introduced as $M_{2}=B_{2} \lambda_{2} B_{2}^{t}$ and $B_{2}^{t}=B_{2}^{-1}$. Equation 14 and 15 also multiply with the matrix on the left $B_{2}^{t}$ and by introducing designation

$$
\begin{gathered}
B_{2}^{t} \theta_{1, i, j}^{(1)}=\theta_{1, i, j}^{(2)} ; \quad B_{2}^{t} \theta_{2, i, j}^{(1)}=\theta_{2, i, j}^{(2)} \text { we get: } \\
\frac{1}{\Delta t} \theta_{1, i, j}^{(2)}+\left(w-w_{g}\right) \frac{\partial \theta_{1, i, j}^{(2)}}{\partial z}= \\
=\left(\alpha_{1, i}^{2}+\beta_{1, \mathrm{j}}^{2}\right) \theta_{1, i, j}^{(2)}+\frac{\partial}{\partial z}\left(k \frac{\partial \theta_{1, i, j}^{(2)}}{\partial z}\right)+ \\
+\delta_{i j} Q_{1, i, j}^{(2)}+F_{1, i, j}^{(2)}+\Phi_{1, i, j}^{(2)}+\frac{1}{\Delta t} \bar{\theta}_{1, i, j}^{(2)} ; \\
\quad \frac{1}{\Delta t} \theta_{2, i, j}^{(2)}+\left(w-w_{g}\right) \frac{\partial \theta_{2, i, j}^{(2)}}{\partial z}= \\
=\left(\alpha_{2, i}^{2}+\beta_{2, j}^{2}\right) \theta_{2, i, j}^{(2)}+\frac{\partial}{\partial z}\left(k \frac{\partial \theta_{2, i, j}^{(2)}}{\partial z}\right)+ \\
+\delta_{i j} Q_{2, i, j}^{(2)}+F_{2, i, j}^{(2)}+\Phi_{2, i, j}^{(2)}+\frac{1}{\Delta t} \bar{\theta}_{2, i, j}^{(2)} .
\end{gathered}
$$

Here

$$
\begin{gathered}
\beta_{j}^{2}=\frac{\alpha_{2, k} \mu_{1}}{h_{y}{ }^{2}} ; \alpha_{2, k}=-2\left(1-\cos \frac{i \pi}{N_{1}+1}\right) ; \\
b_{2, i, j}=(-1) \sqrt[i+j]{\frac{2}{N_{2}+1}} \sin \frac{i j \pi}{N_{2}+1}, \\
\left(i, j=1,2, \ldots ., N_{2}\right) .
\end{gathered}
$$

After some remaking for marginal conditions (5) and (7) we get

$$
\begin{aligned}
& k \frac{\partial \theta_{1, i, j}^{(2)}}{\partial z}=\eta_{1} \theta_{1, i, j}^{(2)}-\Phi_{1, i, j}^{(2)} \\
& k \frac{\partial \theta_{2, i, j}^{(2)}}{\partial z}=\eta_{2} \theta_{2, i, j}^{(2)}-\Phi_{2, i, j}^{(2)}
\end{aligned}
$$

Here $\Phi_{1, i, j}^{(2)}$ and $\Phi_{2, i, j}^{(2)}$ calculates with these formulas

$$
\begin{aligned}
& \Phi_{1, i, j}^{(2)}=\left\{\begin{array}{cc}
U \frac{\theta_{1, i, j}^{(2)}-\theta_{1, i-1, j}^{(2)}}{h_{x}} n p u & U \geq 0 \\
U \frac{\theta_{1, i+1, j}^{(2)}-\theta_{1, i, j}^{(2)}}{h_{x}} n p u & U<0
\end{array}+\right. \\
& +\left\{\begin{array}{l}
V \frac{\theta_{1, i, j}^{(2)}-\theta_{1, i, j-1}^{(2)}}{h_{y}} n p u \quad V \geq 0 \\
V \frac{\theta_{1, i, j+1}^{(2)}-\theta_{1, i, j}^{(2)}}{h_{y}} n p u \quad V<0
\end{array} ;\right. \\
& \Phi_{2, i, j}^{(2)}=\left\{\begin{array}{ll}
U \frac{\theta_{2, i, j}^{(2)}-\theta_{2, i-1, j}^{(2)}}{h_{x}} \text { npu } & U \geq 0 \\
U \frac{\theta_{2, i+1, j}^{(2)}-\theta_{2, i, j}^{(2)}}{h_{x}} \text { npu } & U<0
\end{array}+\right. \\
& +\left\{\begin{array}{l}
V \frac{\theta_{2, i, j}^{(2)}-\theta_{2, i, j-1}^{(2)}}{h_{y}} n p u \quad V \geq 0 \\
V \frac{\theta_{2, i, j+1}^{(2)}-\theta_{2, i, j}^{(2)}}{h_{y}} n p u \quad V<0
\end{array} .\right.
\end{aligned}
$$

Uniting obtained equations (16), (17) and corresponding them marginal conditions usual differential equations are obtained at the last results, which describe the process of moving and diffusion harmful substances to atmosphere by vertical direction, relatively variable $\mathrm{z}$

For the final numerical integration problems the network by variable $\mathrm{z}$ is introduced

$$
\omega_{z}=\left(z_{k}=z_{k-1}-h_{z} ; k=2,3,4,5,6\right),
$$

replacing with differential operators of the differential equations by varieties

$$
\begin{gathered}
\left(\frac{\partial \theta_{1, i, j}^{(2)}}{\partial z}\right)_{\xi}=\left\{\begin{array}{l}
\frac{\theta_{1, i, \mathrm{j}, \xi-1}^{(2)}-\theta_{1, i, j, \xi}^{(2)}}{h z}, n p u\left(W-W_{g}\right) \geq 0 \\
\frac{\theta_{1, i, j, \xi+1}^{(2)}-\theta_{1, i, j, \xi}^{(2)}}{h z}, n p u\left(W-W_{g}\right)<0
\end{array} ;\right. \\
\left(\frac{\partial \theta_{2, i, j}^{(2)}}{\partial z}\right)_{\xi}=\left\{\begin{array}{l}
\frac{\theta_{2, i, j, \xi-1}^{(2)}-\theta_{2, i, j, \xi}^{(2)}, n p u\left(W-W_{g}\right) \geq 0}{h z} \\
\frac{\theta_{2, i, j, \xi+1}^{(2)}-\theta_{2, i, j, \xi}^{(2)}}{h z}, n p u\left(W-W_{g}\right)<0
\end{array} ;\right. \\
=\left[\begin{array}{c}
K_{\xi+0.5} \theta_{1, i, j, \xi+1}^{(2)}-\left(K_{\xi+0.5}+K_{\xi-0.5}\right) \times \\
\times \theta_{1, i, j, \xi}^{(2)}+K_{\xi-0.5} \theta_{1, i, j, \xi-1}^{(2)}
\end{array}\right]
\end{gathered}
$$




$$
\begin{gathered}
\frac{\mathbb{I}}{\operatorname{II} z}\left(K \frac{\mathbb{I} \theta_{2, i, j}^{(2)}}{\mathbb{I} z}\right)_{\xi}= \\
=\left[\begin{array}{c}
K_{\xi+0.5} \theta_{2, i, j, \xi+1}^{(2)}-\left(K_{\xi+0.5}+K_{\xi-0.5}\right) \times \\
\times \theta_{2, i, j, \xi}^{(2)}+K_{\xi-0.5} \theta_{2, i, j, \xi-1}^{(2)}
\end{array}\right] h_{z}^{2},
\end{gathered}
$$

releasing indexes $i, j$ and classification the same members equation, we get:

$$
\begin{aligned}
& \frac{1}{h_{z}^{2}} k_{\xi+0.5}-\theta_{1, \xi+1}^{(2)}-\frac{1}{h_{z}^{2}}\left(k_{\xi+0.5}+k_{\xi-0.5}\right) \theta_{1, \xi}^{(2)}+ \\
& +\frac{1}{h_{z}^{2}} k_{\xi-0.5}-\theta_{1, \xi-1}^{(2)}+ \\
& \int\left(\frac{\left(w-w_{g}\right)}{h_{z}} \theta_{1, \xi-1}^{(2)}-\frac{\left(w-w_{g}\right)}{h_{z}} \theta_{1, \xi}^{(2)}\right) \\
& n p u\left(w-w_{g}\right) \geq 0 \\
& \left\{\left(\frac{\left(w-w_{g}\right)}{h_{z}} \theta_{1, \xi+1}^{(2)}-\frac{\left(w-w_{g}\right)}{h_{z}} \theta_{1, \xi}^{(2)}\right)^{=}\right. \\
& n p u\left(w-w_{g}\right)<0 \\
& =q_{1} \theta_{1, \xi+1}^{(2)}-q_{2} \theta_{1, \xi}^{(2)}+q_{3} \theta_{1, \xi-1}^{(2)}+ \\
& +\left\{\begin{array}{l}
\left(q_{4}\left(\theta_{1, \xi-1}^{(2)}-\theta_{1, \xi}^{(2)}\right)\right) \text { nрu }\left(w-w_{g}\right) \geq 0 \\
\left(q_{4}\left(\theta_{1, \xi+1}^{(2)}-\theta_{1, \xi}^{(2)}\right)\right) \text { nрu }\left(w-w_{g}\right)<0
\end{array} ;\right. \\
& \frac{1}{h_{z}^{2}} k_{\xi+0.5}-\theta_{2, \xi+1}^{(2)}-\frac{1}{h_{z}^{2}}\left(k_{\xi+0.5}+k_{\xi-0.5}\right) \theta_{2, \xi}^{(2)}+ \\
& +\frac{1}{h_{z}^{2}} k_{\xi-0.5}-\theta_{2, \xi-1}^{(2)}+ \\
& \int\left(\frac{\left(w-w_{g}\right)}{h_{z}} \theta_{2, \xi-1}^{(2)}-\frac{\left(w-w_{g}\right)}{h_{z}} \theta_{2, \xi}^{(2)}\right) \\
& +\left\{n p u\left(w-w_{g}\right) \geq 0\right. \\
& \left\{\left(\frac{\left(w-w_{g}\right)}{h_{z}} \theta_{2, \xi+1}^{(2)}-\frac{\left(w-w_{g}\right)}{h_{z}} \theta_{2, \xi}^{(2)}\right)^{=}\right. \\
& n p u\left(w-w_{g}\right)<0 \\
& =q_{1} \theta_{2, \xi+1}^{(2)}-q_{2} \theta_{2, \xi}^{(2)}+q_{3} \theta_{2, \xi-1}^{(2)}+ \\
& +\left\{\begin{array}{l}
\left(q_{4}\left(\theta_{2, \xi-1}^{(2)}-\theta_{2, \xi}^{(2)}\right)\right) \text { nрu }\left(w-w_{g}\right) \geq 0 \\
\left(q_{4}\left(\theta_{2, \xi+1}^{(2)}-\theta_{2, \xi}^{(2)}\right)\right) \text { nрu }\left(w-w_{g}\right)<0
\end{array} .\right.
\end{aligned}
$$

Now using correlation (22) and (23) instead of (16) and (17) we obtain:

$$
\begin{aligned}
& \frac{1}{\Delta t} \theta_{1, \xi}^{(2)}+q_{1} \theta_{1, \xi+1}^{(2)}-q_{2} \theta_{1, \xi}^{(2)}+q_{3} \theta_{1, \xi-1}^{(2)}+ \\
& +\left\{\begin{array}{l}
\left(q_{4}\left(\theta_{1, \xi-1}^{(2)}-\theta_{1, \xi}^{(2)}\right)\right) \text { nри }\left(w-w_{g}\right) \geq 0 \\
\left(q_{4}\left(\theta_{1, \xi+1}^{(2)}-\theta_{1, \xi}^{(2)}\right)\right) \text { nрu }\left(w-w_{g}\right)<0
\end{array}=\right. \\
& =\left(\alpha_{1, i}^{2}+\beta_{1, \mathrm{j}}^{2}\right) \theta_{1, \xi}^{(2)}+\delta_{\xi} Q_{1, \xi}^{(2)}+ \\
& +F_{1, \xi}^{(2)}+\Phi_{1, \xi}^{(2)}+\frac{1}{\Delta t} \bar{\theta}_{1, \xi}^{(2)} ; \\
& \frac{1}{\Delta t} \theta_{2, \xi}^{(2)}+q_{1} \theta_{2, \xi+1}^{(2)}-q_{2} \theta_{2, \xi}^{(2)}+q_{3} \theta_{2, \xi-1}^{(2)}+ \\
& +\left\{\begin{array}{l}
\left(q_{4}\left(\theta_{2, \xi-1}^{(2)}-\theta_{2, \xi}^{(2)}\right)\right) \text { nри }\left(w-w_{g}\right) \geq 0 \\
\left(q_{4}\left(\theta_{2, \xi+1}^{(2)}-\theta_{2, \xi}^{(2)}\right)\right) \text { nри }\left(w-w_{g}\right)<0
\end{array}=\right. \\
& =\left(\alpha_{2, i}^{2}+\beta_{2, \mathrm{j}}^{2}\right) \theta_{2, \xi}^{(2)}+\delta_{\xi} Q_{2, \xi}^{(2)}+ \\
& +F_{2, \xi}^{(2)}+\Phi_{2, \xi}^{(2)}+\frac{1}{\Delta t} \bar{\theta}_{2, \xi}^{(2)}
\end{aligned}
$$

For attributing marginal conditions by $\mathrm{z}=0$ at the underlying surface of the earth we integrate equations (16) and (17) from 0 to $h_{Z / 2}$ and obtain:

$$
\begin{gathered}
\int_{0}^{h_{\mathrm{z}} / 2}\left[\frac{\partial}{\partial z}\left(k \frac{\partial \theta_{1, i, j}^{(2)}}{\partial z}\right)-\left(w-w_{g}\right) \frac{\partial \theta_{1, i, j}^{(2)}}{\partial z}+\right. \\
\left.+\left(\frac{1}{\Delta t}-\left(\alpha_{1, i}^{2}+\beta_{1, j}^{2}\right)\right) \theta_{1, i, j}^{(2)}\right] d z=\int_{0}^{h_{\mathrm{z}} / 2} \bar{G}_{1, i, j}^{(2)} d z, \\
\quad \int_{0}^{h_{\mathrm{z}} / 2}\left[\frac{\partial}{\partial z}\left(k \frac{\partial \theta_{2, i, j}^{(2)}}{\partial z}\right)-\left(w-w_{g}\right) \frac{\partial \theta_{2, i, j}^{(2)}}{\partial z}+\right. \\
\left.+\left(\frac{1}{\Delta t}-\left(\alpha_{2, i}^{2}+\beta_{2, j}^{2}\right)\right) \theta_{2, i, j}^{(2)}\right] d z=\int_{0}^{h_{\mathrm{z}} / 2} \bar{G}_{2, i, j}^{(2)} d z,
\end{gathered}
$$

where

$$
\begin{aligned}
& \bar{G}_{1, i, j}^{(2)}=\delta_{i, j} Q_{1, i, j}^{(2)}+F_{1, i, j}^{(2)}+\Phi_{1, i, j}^{(2)}+\frac{1}{\Delta t} \bar{Q}_{1, i, j}^{(2)} ; \\
& \bar{G}_{2, i, j}^{(2)}=\delta_{i, j} Q_{2, i, j}^{(2)}+F_{2, i, j}^{(2)}+\Phi_{2, i, j}^{(2)}+\frac{1}{\Delta t} \bar{Q}_{2, i, j}^{(2)} .
\end{aligned}
$$

Taking on account marginal conditions equations (26) and (27) we can write as:

$$
\begin{gathered}
\int_{0}^{h_{z} / 2} \frac{\partial}{\partial z}\left(k(z) \frac{\partial \theta_{1, i, j}^{(2)}}{\partial z}\right) d z= \\
=\left.k\left(z_{1 / 2}\right) \frac{\partial \theta_{1, i, j}^{(2)}}{\partial z}\right|_{1 / 2}-\left.k\left(z_{1 / 2}\right) \frac{\partial \theta_{1, i, j}^{(2)}}{\partial z}\right|_{0}= \\
=k\left(z_{1 / 2}\right) \frac{\theta_{1, i, j, 2}^{(2)}-\theta_{1, i, j, 1}^{(2)}}{h_{z}}-\eta_{1} \theta_{1, i, j, 1}^{(2)},
\end{gathered}
$$




\begin{tabular}{l|lrl|l|ll} 
& ISRA (India) & $=\mathbf{1 . 3 4 4}$ & SIS (USA) & $=\mathbf{0 . 9 1 2}$ & ICV (Poland) & $=\mathbf{6 . 6 3 0}$ \\
Impact Factor: & ISI (Dubai, UAE) $=\mathbf{0 . 8 2 9}$ & PUHЦ (Russia) & $=\mathbf{0 . 2 3 4}$ & PIF (India) & $=\mathbf{1 . 9 4 0}$ \\
& GIF (Australia) & $\mathbf{0 . 5 6 4}$ & ESJI (KZ) & $=3.860$ & IBI (India) & $=\mathbf{4 . 2 6 0}$ \\
& JIF & $\mathbf{1 . 5 0 0}$ & SJIF (Morocco) & $\mathbf{2 . 0 3 1}$ & & \\
\hline
\end{tabular}

$$
\begin{gathered}
\int_{0}^{h_{z} / 2}\left(w-w_{g}\right) \frac{\partial \theta_{1, i, j}^{(2)}}{\partial z} d z= \\
=\left(w-w_{g}\right)_{1 / 4}\left(\theta_{1, i, j, 1 / 2}^{(2)}-\theta_{1, i, j, 0}^{(2)}\right)= \\
=\left(w-w_{g}\right)_{1 / 4} \frac{\theta_{1, i, j, 1}^{(2)}-\theta_{1, i, j, 0}^{(2)}}{2}-\theta_{1, i, j, 0}^{(2)}= \\
=\left(w-w_{g}\right)_{1 / 4} \frac{1}{2}\left(\theta_{1, i, j, 0}^{(2)}-\theta_{1, i, j, 1}^{(2)}\right), \\
\int_{0}^{h_{z} / 2} \bar{G}_{1, i, j}^{(2)} d z=\bar{G}_{0}^{(2)} \frac{h_{z}}{2}, \\
k\left(z_{1 / 2}\right)_{1,2}^{(2)}-\theta_{1,1}^{(2)}-\eta_{1} \theta_{1,1}^{(2)}- \\
-\left(w-w_{g}\right)_{1 / 4} \frac{1}{2}\left(\theta_{1,0}^{(2)}-\theta_{1,1}^{(2)}\right)- \\
-\left(\frac{1}{\Delta t}-\left(\alpha_{1, i}^{2}+\beta_{1, j}^{2}\right)\right) \theta_{1,1}^{(2)}=\bar{G}_{i, j, 0}^{2}
\end{gathered}
$$

or

$$
\begin{gathered}
2 k\left(z_{1 / 2}\right)\left(\theta_{1,2}^{(2)}-\theta_{1,1}^{(2)}\right)-2 h_{z} \eta_{1} \theta_{1,1}^{(2)}- \\
-\left(w-w_{g}\right)_{1 / 4} h_{z}\left(\theta_{1,0}^{(2)}-\theta_{1,1}^{(2)}\right)- \\
-2 h_{z}\left(\frac{1}{\Delta t}-\left(\alpha_{1, i}^{2}+\beta_{1, j}^{2}\right)\right) \theta_{1,1}^{(2)}=2 h_{z} \bar{G}_{i, j, 0}^{2} .
\end{gathered}
$$

Than at last we get:

$$
\begin{gathered}
2 k\left(z_{1 / 2}\right) \theta_{1, i, j, 2}^{(2)}-\left[2 K\left(z_{1 / 2}\right)+2 h_{z} \eta_{1}+\left(w-w_{g}\right) h_{z}+\right. \\
\left.+2 h_{z}\left(\frac{1}{\Delta t}-\left(\alpha_{1, i}^{2}+\beta_{1, j}^{2}\right)\right)\right] \theta_{1, i, j, 1}^{(2)}- \\
-\left(w-w_{g}\right) h_{z} \theta_{1, i, j, 0}^{(2)}=2 h_{z} \bar{G}_{1, i, j, 0}^{(2)},
\end{gathered}
$$

and for $\theta_{2, i, j, \zeta}^{(2)}$

$$
\begin{gathered}
2 k\left(z_{1 / 2}\right) \theta_{2, i, j, 2}^{(2)}-\left[2 k\left(z_{1 / 2}\right)+2 h_{z} \eta_{2}+\left(w-w_{g}\right) h_{z}+\right. \\
\left.+2 h_{z}\left(\frac{1}{\Delta t}-\left(\alpha_{2, i}^{2}+\beta_{2, j}^{2}\right)\right)\right] \theta_{2, i, j, 1}^{(2)}- \\
-\left(w-w_{g}\right) h_{z} \theta_{1, i, j, 0}^{(2)}=2 h_{z} \bar{G}_{2, i, j, 0}^{(2)} .
\end{gathered}
$$

In addition integrating equations (16) and (17) from $(N+1 / 2) h_{z}$ to $(N+1) h_{z}$ we get:

$$
\begin{gathered}
\int_{G_{H}-1 / 2 h_{z}}^{G_{H}} \frac{\partial}{\partial z}\left(k(z) \frac{\partial \theta_{1}^{(2)}}{\partial z}\right) d z= \\
=\left[k\left(z_{G_{H}}\right) \frac{\partial \theta_{1, i, j, N}^{(2)}}{\partial z}-k\left(z_{G_{H}-1 / 2 h_{z} N}\right) \frac{\partial \theta_{1, i, j, H-1 / 2 h_{z} N}^{(2)}}{\partial z}\right]= \\
=k\left(Z_{1 / 2}\right) \frac{\theta_{1, i, j, N+1}^{(2)}-\theta_{1, i, j, N}^{(2)}}{h_{z}}= \\
=k\left(Z_{G_{H}-1 / 2 h_{z}}\right) / h_{z} \theta_{1, i, j, N}^{(2)}+k\left(Z_{G_{H}-1 / 2 h_{z}}\right) / h_{z} \theta_{1, i, j, N+1}^{(2)} ; \\
\int_{G_{H}}^{G_{H}}\left(w-w_{g}\right) \frac{\partial \theta_{1}^{(2)}}{\partial z} d z= \\
=\left(w-w_{g}\right)_{z_{G_{H}-1 / 2 h^{h_{z}}}}\left(\theta_{1, i, j, N-1}^{(2)}-\theta_{1, i, j, N+0.5}^{(2)}\right) .
\end{gathered}
$$

So by $z=G_{H}$ we get :

$$
\begin{gathered}
\frac{k\left(z_{G_{H}-1 / h_{z}}\right)}{h_{z}} \theta_{1, i, j, N}^{(2)}+\frac{k\left(z_{G_{H}-1 / h_{z}}\right)}{h_{z}} \theta_{1, i, j, N+1}^{(2)}+ \\
+\left(\frac{1}{\Delta t}-\left(\alpha_{1, i}^{2}+\beta_{1, j}^{2}\right)\right) \theta_{1, i, j, N}^{(2)}- \\
-\left(w-w_{g}\right)_{z_{G_{H}-1 / h^{h}}}\left(\theta_{1, i, j, N-1}^{(2)}-\theta_{1, i, j, N+0.5}^{(2)}\right)=G_{1, i, j, N}^{(2)}
\end{gathered}
$$

or

$$
\begin{gathered}
\gamma_{1} \theta_{1, i, j, N}^{(2)}+\gamma_{2} \theta_{1, i, j, N+1}^{(2)}+\gamma_{3} \theta_{1, i, j, N}^{(2)}-\gamma_{4} \theta_{1, i, j, N-1}^{(2)}- \\
-\gamma_{4} \frac{\theta_{1, i, j, N}^{(2)}+\theta_{1, i, j, N+1}^{(2)}}{2}=G_{1, i, j, N}^{(2)}
\end{gathered}
$$

at last

$$
\begin{gathered}
\left(\gamma_{2}+\gamma_{4} / 2\right) \theta_{1, i, j, N+1}^{(2)}+\left(\gamma_{1}+\gamma_{3}+\gamma_{4} / 2\right) \times \\
\times \theta_{1, i, j, N}^{(2)}+-\gamma_{4} \theta_{1, i, j, N-1}^{(2)}=G_{1, i, j, N}^{(2)} .
\end{gathered}
$$

Here

$$
\begin{gathered}
\gamma_{1}=\frac{k\left(z_{G_{H}-1 / 2 h_{z}}\right)}{h_{z}} ; \gamma_{2}=\frac{k\left(z_{G_{H}-1 / 4 h_{z}}\right)}{h_{z}} ; \\
\gamma_{3}=\left(\frac{1}{\Delta t}-\left(\alpha_{1, i}^{2}+\beta_{1, j}^{2}\right)\right) ; \gamma_{4}=\left(w-w_{g}\right)_{z_{G_{H}-1 / h_{z}}} .
\end{gathered}
$$

Analogous for $\theta_{2, i, j}^{(2)}$ at last we get expression

$$
\begin{gathered}
\left(\gamma_{2}+\gamma_{4} / 2\right) \theta_{2, i, j, N+1}^{(2)}+\left(\gamma_{1}+\gamma_{3}+\gamma_{4} / 2\right) \times \\
\times \theta_{2, i, j, N}^{(2)}-\gamma_{4} \theta_{2, i, j, N-1}^{(2)}=G_{2, i, j, N}^{(2)} .
\end{gathered}
$$

So, finally for decision $\theta_{1, i, j, \xi}^{(2)}$ and $\theta_{2, i, j, \xi}^{(2)}$ we obtain the system algebraical equations 


\begin{tabular}{|c|c|c|c|c|c|c|}
\hline Impact Factor: & $\begin{array}{l}\text { ISRA (India) } \\
\text { ISI (Dubai, UAE } \\
\text { GIF (Australia) } \\
\text { JIF }\end{array}$ & $\begin{array}{l}=1.344 \\
=0.829 \\
=0.564 \\
=1.500\end{array}$ & $\begin{array}{l}\text { SIS (USA) } \\
\text { PИНЦ (Russia) } \\
\text { ESJI (KZ) } \\
\text { SJIF (Morocco) }\end{array}$ & $\begin{array}{l}=0.912 \\
=0.234 \\
=3.860 \\
=\mathbf{2 . 0 3 1}\end{array}$ & $\begin{array}{l}\text { ICV (Poland) } \\
\text { PIF (India) } \\
\text { IBI (India) }\end{array}$ & $\begin{array}{l}=6.630 \\
=1.940 \\
=4.260\end{array}$ \\
\hline
\end{tabular}

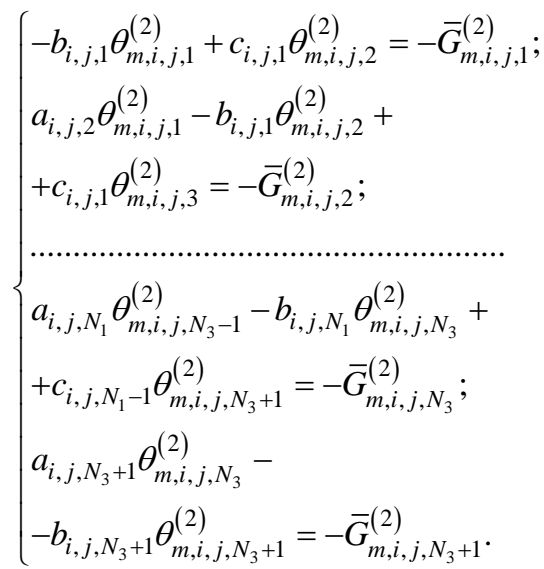

Where accept sense $m=1,2$.

Solving the problem (28), we find $\theta_{1, i, j, \xi}^{(2)}$, $\theta_{2, i, j, \xi}^{(2)} \quad$ by $\quad i=0,1,2, \ldots, N_{1}+1 ; \quad j=0,1,2, \ldots, N_{2}$; $\xi=0,1,2, \ldots, N_{3}$. then with correlations (16), (17) and by quest sense $\theta_{1, i, j, \xi}^{(2)}, \theta_{2, i, j, \xi}^{(2)} \theta_{1, i, j, \xi}^{(1)}, \theta_{2, i, j, \xi}^{(1)}$. With (12), (13) by $\theta_{1, i, j, \xi}^{(1)}, \theta_{2, i, j, \xi}^{(1)}$ we cross to numerical solving problem (8) and (9) at the momnt of time

\section{Conclusion}

To conduct a comprehensive study of the propagation of harmful substances into the environment, taking into account the mutual conversion of aerosol particles in the atmosphere due to changes in climatic factors, a mathematical model was developed, more adequately describing the object of study.

The differential equation for the calculation of the fine and the rate of deposition of the aerosol particles propagating in the boundary layer of the atmosphere, which takes into account the mass and radius of the aerosol particle density of the atmosphere, the force of air resistance.

For the numerical solution of the problem we developed an efficient algorithm based on the "direct method", which allows to reduce the multidimensional problem described by differential equations in partial derivatives, to the integration of ordinary differential equation.

\section{References:}

1. Suxinov AI, Xachuns DS (2013) Programmnaya realizasiya dvumernoy zadachi dvijeniya vozdushnoy sredi // Izvestiya Yujnogo federalnogo universiteta. Texnicheskiye nauki. 2013. № 4. p. 15-20.

2. Chistyakov AY, Xachuns DS (2013) Zadacha dvijeniya mnogokomponentnoy vozdushnoy sredi s uchetom paroobrazovaniya i kondensasii // Izvestiya Yujnogo federalnogo universiteta. Texnicheskiye nauki. 2013. № 4. p. 87-98.

3. Izrael YA, Nazarov IM, Pressman AY, Rovinskiy FY, Ryaboshapko AG, Filippova LM (1983) Kislotniye dojdi. L.: Gidrometeoizdat, 206 p.

4. Anderson GE (1971) Mesoscale influences on wind fields. J. Appl. Meteor., 1971, 10, p. 377386.

5. Fulton SR, Schubert WH (1987) Chebyshev spectral methods for limited- area models, Part I. Model problem analysis. Mon. Wea. Rev., 1987, No. 115, p. 1940- 1953.

6. Huber AH, Snyder WH (1976) Building Wake effects on Short Stack Effluents. Preprint Volume for Triad Symposium Atmospheric Diffusion and Air Quality. American Meteorological Society, Boston, MA.
7. Vizova NL, Garger YK, Ivanov VN (1991) Eksperimentalniye issledovaniya atmosfernoy diffuzii i rascheti rasseyaniya primesi. Leningrad, Gidrometeoizdat, 278 p.

8. Danilov AD (2014) Matematicheskoye modelirovaniye dinamiki fizicheskix prosessov v atmosfere / A. D. Danilov, A. V. Pileich // Aktualnыye napravleniya nauchnix issledovaniy XXI veka: teoriya i praktika. 2014. T. 2. №. 4, p.2. p.288-291. DOI: $10.12737 / 5167$

9. Penenko A, Sorokovaya K, Sorokovoy A (2015) A numerical model of bio-aerosols transformation in the atmosphere/ Numerical Modeling in Atmosphere, Ocean, and Environment Studies The Bulletin of NCC, - p. 37-50.

10. Perepechko $\mathrm{Y}$, Sorokin $\mathrm{K}$, Imomnazarov $\mathrm{K}$ (2017) Modeling of the nonlinear motion of saturated granular media Numerical Modeling in Atmosphere, Ocean, and Environment Studies The Bulletin of NCC. p. 51-65.

11. Xolmatjanov BM, Petrov YV, Fatxullayeva ZN (2000) Trexmernaya chislennaya model dlya osenki zagryazneniya atmosferi Axangaranskoy dolini promishlennimi vibrosami sernistogo angidrida (SO2) i soyedineniyami mishyaka 


\begin{tabular}{l|lrl|l|ll} 
& ISRA (India) & $=\mathbf{1 . 3 4 4}$ & SIS (USA) & $=\mathbf{0 . 9 1 2}$ & ICV (Poland) & $=\mathbf{6 . 6 3 0}$ \\
Impact Factor: & ISI (Dubai, UAE) $=\mathbf{0 . 8 2 9}$ & PUHL (Russia) $=\mathbf{0 . 2 3 4}$ & PIF (India) & $=\mathbf{1 . 9 4 0}$ \\
& GIF (Australia) & $\mathbf{0 . 5 6 4}$ & ESJI (KZ) & $=3.860$ & IBI (India) & $=\mathbf{4 . 2 6 0}$ \\
& JIF & $\mathbf{1 . 5 0 0}$ & SJIF (Morocco) & $=\mathbf{2 . 0 3 1}$ & & \\
\hline
\end{tabular}

(As) // Vestnik TashGU. - Tashkent, 2000. №3. - p. 19-25.

12. Petrov YV, Xolmatjanov BM, Fatxullayeva ZN (2000) Lokalnaya gidrodinamicheskaya model i raschetnaya sxema perenosa zagryaznyayushix veshestv $\mathrm{v}$ atmosfere // Ispolzovaniye sovremennix metodov analiza dlya resheniya geofizicheskix zadach: Tez. dokl. Resp. konf. 18-20 oct. 2000. - Tashkent: SANIGMI, 2000. p. $57-58$.

13. Kholmatjanov BM (2002) Application of Three-Dimensional Model for Estimation of Atmosphere Pollution on Condition MountainValley Circulation // Bulliten of the Novosibirsk Computing Center. Series "Numerical modeling in Atmosphere, Ocean and Environment Studies", ICM MG Publisher. Novosibirsk, 2002. Issue 8. - p. 19-29.

14. Xolmatjonov BM (2003) Osenka zagryazneniya atmosferi Axangaranskoy dolini v period fyona s pomoshyu gidrodinamicheskoy modeli // Sb. nauch. trudov. - Tashkent: SANIGMI. - p. 2939.

15. Xolmatjanov BM (2004) Raschet poley zagryazneniya atmosferi Axangaranskoy dolini $\mathrm{v}$ period fyona s pomoщyu gidrodinamicheskoy modeli // ENVIROMIS-2004: Materiali mejdunarodnoy konferensii. Tomsk, 17-25 iyulya 2004. - p. 73-74.

16. Xolmatjanov BM, Fatxullayeva ZN (2005) Modelirovaniye, raschet i prognoz perenosa zagryaznyayushix veshyestv v Axangaranskoy promishlennoy zone Uzbekistana // Ekologicheskiye i gidrometeorologicheskiye problemi bolshix gorodov i promishlennыx zon: Materiali mejdunarodnoy nauchnoy konferensii. Sankt-Peterburg, 25-27 maya 2005. - SanktPeterburg: RGGMU, 2005. - p. 38.
17. Xolmatjanov BM, Fatxullayeva ZN, Petrov YV, Egamberdiyev XT (2008) Tipi sinopticheskix prosessov Sredney Azii, obuslavlivayushiye mestniye sirkulyasii $\mathrm{v}$ dolinax Zapadnogo Tyan-Shanya // Tr. Instituta Gidrometeorologii Gruzii. - Tbilisi, 2008. - Tom №115. - p. 195203.

18. Guniya GS, Skvitinidze ZI, Xolmatjanov BM, Fatxullayeva ZN (2008) K meteorologicheskim aspektam krupnomasshtabnix perenosov zagryaznyayushix veshestv $\mathrm{v}$ usloviyax gornix regionov // Tr. Instituta Gidrometeorologii Gruzii. - Tbilisi, 2008. - Tom №115. - p. 266277.

19. Ravshanov N, Shertaev M, Toshtemirova N (2015) Mathematical Model for the Study and Forecast of the Concentration of Harmful Substances in the Atmosphere // American Journal of Modeling and Optimization. - 2015. - Vol. 3. - № 2. - p. 35-39.

20. Ravshanov N, Sharipov DK, Axmedov D (2015) Modelirovaniya prosessa zagryazneniya okrujayushey sredi s uchetom relyefa mestnosti pogodno-klimaticheskix faktorov // Informasionniye texnologii modelirovaniya $\mathrm{i}$ upravleniya - Voronej, 2015.№3. - p. 222-235.

21. Sharipov DK (2013) Development of mathematical software aerosol transport and diffusion of the atmospheric emissions // European Applied Sciences. - 2013. -Vol. 1. № 1. - pp. 233-240.

22. Faddeyeva VN (1949) Metod pryamыx v primenenii $\mathrm{k}$ nekotorыm krayevыm zadacham//Trudы Matematicheskogo instituta AN SSSR im. V.A. Steklova, t. XXVIII (28), M.. 